\title{
SITUATIONS IN CONSTRUCTION OF 3D MAPPING FOR SLAM
}

\author{
Trang Nguyen Hoang Thuy ${ }^{1,2^{*}}$, Stanislav Shydlouski ${ }^{2}$ \\ ${ }^{1}$ National Research Tomsk State University, 634050, Tomsk, Russia \\ ${ }^{2}$ Vietnam Maritime University, 484 Lach Tray, Le Chan, Hai Phong, Viet Nam
}

\begin{abstract}
Nowadays, the simultaneous localization and mapping (SLAM) approach has become one of the most advanced engineering methods used for mobile robots to build maps in unknown or inaccessible spaces. Update maps before a certain area while tracking current location and distance. The motivation behind writing this paper is mainly to help us better understand about SLAM and the study situation of SLAM in the world today. Through this, we find the optimal algorithm for moving robots in three dimensions.
\end{abstract}

\section{Introduction}

Along with the development of science and technology, in robotic mapping and navigation has been studied and more interested by scientists. For the purpose of fixing and improving positioning in short distances, simultaneous localization and mapping (SLAM) has marked significant advances in the development of science and technology. Previously, thanks to GPS technology (Global Positioning System) it is easy to pinpoint directions or give a route for the journey, now SLAM technology is also moving closer to human life with certain uses. It helps people to locate oneself and create a model of your surroundings, which is important in many applications. Thanks to the simultaneous localization and mapping (SLAM) technology, positioning approach commonly used indoors or underground where satellite navigation (GPS) is not viable because the enclosed space and the distance are too small under 6 meters. Research on SLAM has been started in the robotics community since 1986 in the papers of Smith and Cheeseman, sually with wheeled robots traversing a flat ground plane. Typically this was done by combining sensor readings (such as from a laser scanner) information about the control input (eg, steering angle) and the measured robot state (eg. counting wheel rotations). This could be a core difficulty of SLAM, such as creating a consistent and accurate map, making the best use of multiple unreliable sources of information [1]. Popular SLAM applications are home robots, unmanned aircraft, self-driving cars.

\footnotetext{
* Corresponding author: trangnht@vimaru.eud.vn
} 


\section{The concept of SLAM, localization and mapping}

SLAM estimates the pose of a robot and the map of the environment at the same time. SLAM is hard by two main reasons. The first, a map is needed for localization (inferring location given a map), the second a good pose estimate is needed for mapping (inferring a map given locations).

SLAM: learning a map and locating the robot simultaneously [2].

SLAM technology was born in the robotics industry and is used by autonomous vehicles to concurrently map and navigate through an unknown environment. To do this, SLAM algorithms utilise information from sensors (often Lidar or imagery) to compute a best estimate of the device's location and a map of the environment around it.

SLAM algorithms are tailored to the available resources, hence not aimed at perfection, but at operational compliance. Published approaches are employed in self-driving cars, unmanned aerial vehicles, autonomous underwater vehicles, planetary rovers, newly emerging domestic robots and even inside the human body [3].

\section{So what are the benefits of Geospatial SLAM?}

The main advantage of Geospatial SLAM for 3D mapping applications is that scanning can be undertaken whilst mobile, and without the need for GPS. This facilitates rapid and accurate 3D acquisition of complex environments and is particularly effective for indoor mapping or surveys of enclosed environments that would not be possible using current GPS based mobile mapping solutions. When compared to traditional survey methods for measuring indoors, such as tape and Disto, or even static laser scanning, mobile indoor mapping can prove to be up to 10 times faster [4].

From 2014, Google has worked on mapping systems for in-house 3D mapping. This system includes both proprietary 3D hardware and software that processes the sensor data into the map. In 2016 Google released the Lidar SLAM real-time library. The SLAM algorithm uses LIDAR and IMU data to simultaneously identify the location of the sensor and create a tight map around it. But the solution will also have strengths and weaknesses so the company has plans to add new solutions to overcome the shortage in the existing SLAM algorithm that is the solution "Lifelong mapping '. By updating the SLAM algorithm so that an autonomous device can estimate the likelihood that a feature in its environment is still static or moving [5].

3D Mapping is a technology that allows you to change the visual characteristics of an object using a video projection and is able to turn almost any surface into a dynamic video screen.

SLAM is concerned with the problem of building a map of an unknown environment by a mobile robot while at the same time navigating the environment using the map.

SLAM consists of multiple parts; Landmark extraction, data association, state estimation, state update and landmark update.

SLAM is a key component of any truly autonomous robot. Much recent research has been done tackling the computational efficiency of SLAM and the data association and landmark extraction necessary for a robust SLAM method.

Environmental mapping involves creating a mathematical model of a real environment's spatial information. SLAM extends the requirements of this mathematical model; it must also jointly represent the robot's state and the position of extracted landmarks relative to the robot's location. The robot's state includes information on the robot's position and orientation. The basic SLAM framework involves odometry, landmark prediction, landmark extraction, data as sociation and matching, pose estimation, and map update. These processes are the backbone of every major SLAM method, and are performed in cyclic fashion. 
SLAM algorithms must take account of a variety of parameters i.e. sensors, map representation, robot dynamics, environmental dynamics, and the integration of sensor measurements and the robot's control system over time.

The integration of these diverse parameters is most often accomplished using the two major SLAM-related algorithms. Kalman filters are routinely used, with the most popular being the Extended Kalman Filter (EKF) [6 -8].

Rao-Blackwellized Particle Filters (RBPF) [9, 10] have also been used in many SLAM methods.

Mapping the spatial information of an environment requires spatial sensors to which SLAM algorithms can be applied. The two most popular sensor modalities used in SLAM are raw range scan sensors and feature (landmark) based sensors (whether extracted from scans or images) $[11,12]$.

\section{SLAM Problems}

SLAM is a chicken-or-egg problem: i) a map is needed for localization. ii) a pose estimate is needed for mapping.

SLAM is currently one of the most widely studied, most interested in robotics. The problem of SLAM is clarified as follows. Consider a mobile robot consisting of two wheels connected to the engine, containing a control processor and a camera attached to it. Through the camera, the programmer can see objects around the robot, taking measurements of the distance from the robot to the surrounding objects, and processing the color in three dimensions when identifying the object. Know where there are objects, where is the way. By identifying that, the programmer will program a road map for the robot in three dimensions so that it can avoid the surrounding objects and follow the directions that have been driven so that the path is Robotic programming is optimal Figure 1. This is a concrete example of SLAM (Localization and Concurrent Planning).

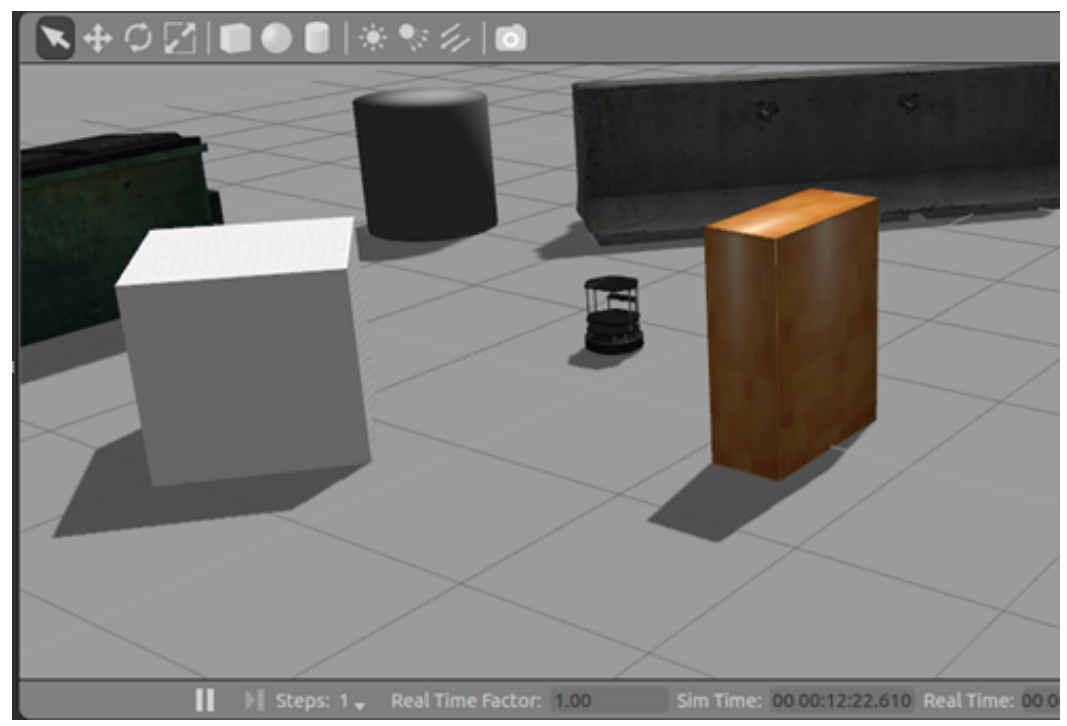

Fig. 1. The robot moves in the gazebo environment.

The environment in which the robot moves is the indoor environment so it will not have the same structure as the pre-loaded robot environment. Robots will be tested in different 
locations to determine the best sensor (Figure 2). The robot recognizes the environment and the programmer creates the most accurate map so that it can move itself in the environment, avoiding the surrounding objects and that will be the optimal path for it.

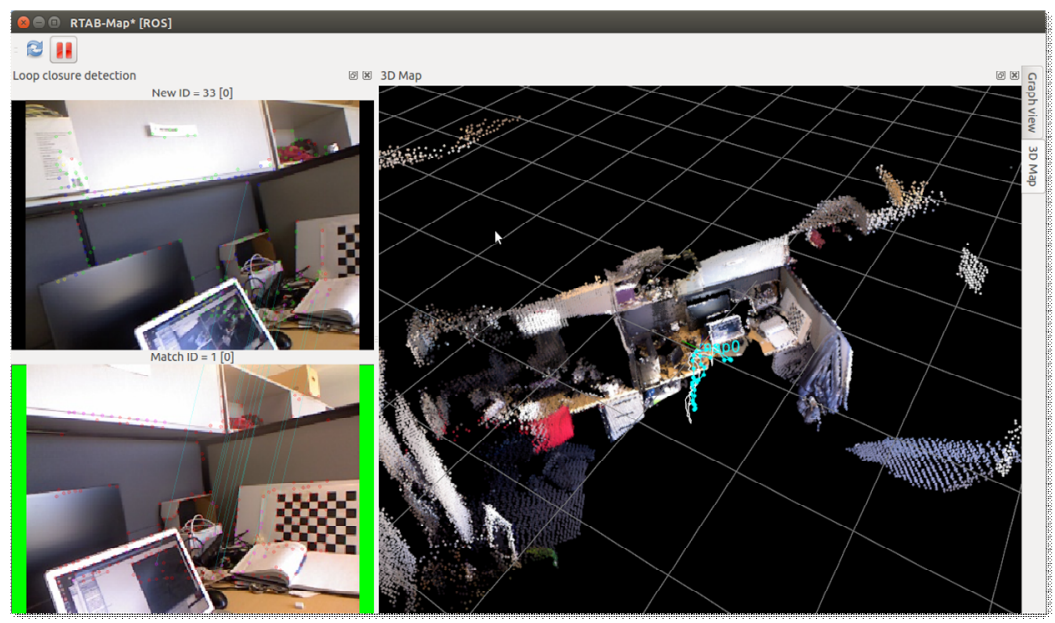

Fig. 2. Robot identifies the environment and creates maps in Rtabmap.

\section{SLAM Applications}

SLAM is central to a range of indoor, outdoor, in-air and underwater applications for both manned and autonomous vehicles (Figure 3). Examples: i) At home: vacuum cleaner, lawn mower; ii) Air: surveillance with unmanned air vehicles; iii) Underwater: reef monitoring; iv) Underground: exploration of mines; v) Space: terrain mapping for localization.

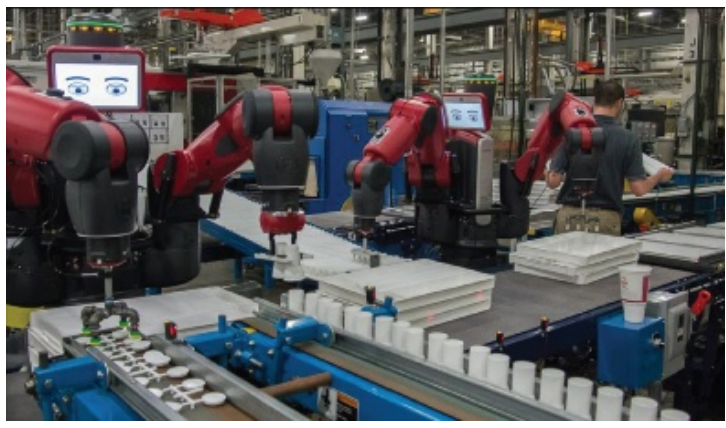

Fig. 3. SLAM technology in the industry.

Autonomous automobiles are now one of the applications of great interest and research. By applying a variety of techniques to detect ambient conditions such as radar, laser light, opacity and computer vision, especially localization and mapping algorithms (SLAM). Selfdriving cars combine data from multiple sensors and off-line maps into current location estimates and map updates (Figure 4). It has simply moved itself with locating the system, real-time positioning (RTLS) and avoids obstructions. 

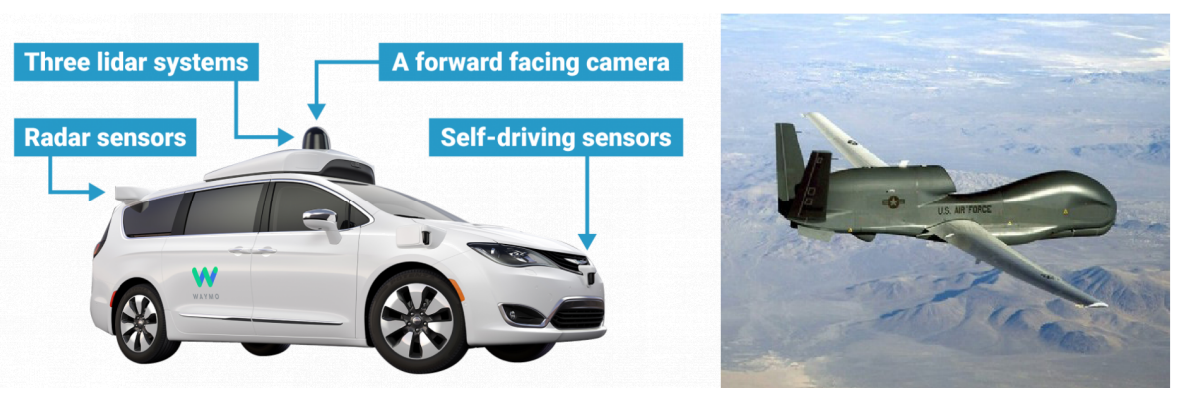

Fig. 4. Self-driving cars and unmanned aircrafts.

This is the topic of simultaneous localization and mapping within the human body (bodySLAM) - Guanqun Bao - in 2014. A Body-SLAM technique that integrates motion information extracted from the video source and RF signal emitted by the capsule to enhance to the localization accuracy of the WCE and meanwhile reconstruct the path the capsule has traveled. The major contribution of this work is that we demonstrated the potential of using endoscopic images to aid the RF localization and possibility to map inside of human body (Figure 5) [13].

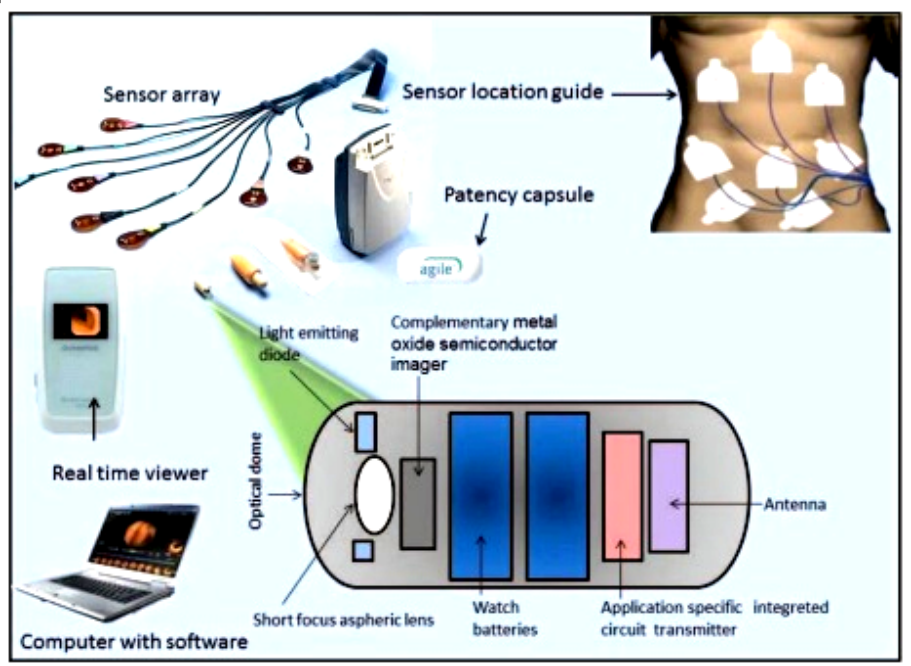

Fig. 5. Typical RF localization system.

Besides, now there are a lot of studies in SLAM:

- 2005 DARPA Grand Challenge winner STANLEY performed SLAM as part of its autonomous driving system.

- SLAM Simultaneous Localisation and Mapping at the Level of Objects [14].

- In 2009: Rafael Valencia, Ernesto H. Teniente, Eduard Trulls, and Juan Andrade-Cetto 3D mapping for urban service robots [15].

- In 2012 Miguel Armando Migueis Pinto: SLAM for 3D Map Building to be used in a Matching Localization Algorithm [16].

- 3D Mapping using Real Earth Stencil (SLAM).

- 3D Mapping with Multimodal Plane SL. 


\section{Conclusion}

The problem of simultaneous localization and mapping has seen great progress over the last 30 years. SLAM and related technologies are increasingly deployed in a variety of settings and practical applications. Slam technology will increasingly be relied upon to provide reliable metric positioning in situations where infrastructure-based solutions such as GPS are unavailable or fail to provide accurate, complete data. SLAM still constitutes an indispensable backbone for most robotics applications and despite the amazing progress over the past decades, existing SLAM systems are far from providing insightful, actionable, and compact models of the environment, comparable to the ones effortlessly created and used by humans.

\section{References}

[1] Osian Haines, An Introduction to Simultaneous Localisation and Mapping, URL https://www.kudan.eu/kudan-news/an-introduction-to-slam/ (2016).

[2] Wolfram Burgard, Cyrill Stachniss, Kai Arras, Maren Bennewitz, Introduction to Mobile Robotics, SLAM: Simultaneous Localization and Mapping, University of Freiburg (2012)

[3] P. Mountney, D. Stoyanov, A. Davison, A. Yang, Simultaneous Stereoscope Localization and Soft-Tissue Mapping for Minimal Invasive Surgery, MICCAI. Lecture Notes in Computer Science. ISBN 978-3-540-44707-8, 347-354 (2006)

[4] John Allan, Mark Reid, Ric Durrant, Geospatial SLAM, URL https://geoslam.com/slam (2012)

[5] Sean Higgins, Google Releases LiDAR SLAM Algorithms, Teases Innovative Mapping Solution, URL https://www.spar3d.com/news/software/google-releases-lidar-slamalgorithms-teases-innovative-mapping-solution/, Spar 3D editor, (2016)

[6] H. Wang, J. Wang, Le Yu, L. Zhen-ye, A new SLAM method based on SVM-AEKF for $A U V$. In OCEANS, 1-6. IEEE (2011)

[7] J. Il-Kyun, S. Lacroix, High resolution terrain mapping using low attitude aerial stereo imagery, in Computer Vision, proceedings. 9th IEEE Conf., 946-951, (2003)

[8] T. Suzuki, Y. Amano, T. Hashizume, Development of a sift based monocular EKF-SLAM algorithm for a small unmanned aerial vehicle, in SICE Annual Conf., Proceedings of IEEE, 1656-1659 (2011)

[9] M. Montemerlo, S. Thrun, D. Koller, B. Wegbreit, in AAAI/IAAI, 593 (2002)

[10]X. Li, W. Cui, J. S.Jia, EEE Conf., 779 (2010)

[11]H. Lategahn, A. Geiger, and B. Kitt, IEEE Conf., 1732 (2011)

[12] T.J. Chong, X.J. Tang, C.H. Leng, M. Yogeswaran, O.E. Ng, Y.Z. Chong, Sensor Technologies and Simultaneous Localization and Mapping (SLAM) (Universiti Tunku Abdul Rahman, Malaysia, 2015)

[13] Guanqun Bao, On Simultaneous Localization and Mapping inside the Human Body (Body-SLAM), (2014)

[14] R.F. Salas-Moreno, R.A. Newcombe, H. Strasdat, P.H.J. Kelly, A.J. Davison, SLAM++: Simultaneous Localisation and Mapping at the Level of Objects (Imperial College London, University of Washington, 2013)

[15]R. Valencia, E.H. Teniente, E. Trulls, J.A. Cetto, 3D Mapping for Urban Service Robots, (2009)

[16] M.A.M. Pinto, SLAM for 3D Map Building to be used in a Matching Localization Algorithm, (2012). 